\title{
RESEARCH
}

Open Access

\section{Cadherin-12 contributes to tumorigenicity in colorectal cancer by promoting migration, invasion, adhersion and angiogenesis}

Jingkun Zhao ${ }^{\dagger}$, Pu Li ${ }^{\dagger}$, Hao Feng, Puxiongzhi Wang, Yaping Zong, Junjun Ma, Zhuo Zhang, Xuehua Chen, Minhua Zheng, Zhenggang Zhu* and Aiguo Lu*

\begin{abstract}
Background: Cadherin 12 (CDH12), which encodes a type II classical cadherin from the cadherin superfamily, may mediate calcium-dependent cell adhesion. It has been demonstrated that $\mathrm{CDH} 12$ could play an important role in the invasion and metastasis of salivary adenoid cystic carcinoma. We decided to investigate the relationship between $\mathrm{CDH} 12$ expression level and clinicopathologic variables in colorectal carcinoma (CRC) patients and to explore the functions of $\mathrm{CDH} 12$ in tumorigenesis in CRC.

Methods: The expression levels of $\mathrm{CDH} 12$ in colorectal carcinoma tissues were detected by immunohistochemistry. Real-time PCR and Western Blot were used to screen CDH12 high-expression cell lines. CCK-8 assay was used to detect the proliferation ability of CRC cells being transfected by shRNAs against $\mathrm{CDH12}$. The wound assay and transwell assay were performed to test migration and invasion ability. The importance of $\mathrm{CDH} 12$ in cell-cell junctions was detected by cell adhesion assay and cell aggregation assay. Endothelial tube formation assay was used to test the influence of $\mathrm{CDH} 12$ on angiogenesis.

Results: Statistical analysis of clinical cases revealed that the positive rate of $\mathrm{CDH} 12$ was higher in the CRC tumor tissues compared with the adjacent non-tumor tissues. The expression levels of $\mathrm{CDH} 12$ in CRC patients are significantly correlated with invasion depth. Consistently, the ability of proliferation, migration and invasion were suppressed when $\mathrm{CDH} 12$ was decreased in CRC cells transfected with shRNAs. Cell adhesion assay and cell aggregation assay presented that tumor cells tend to disperse with the lack of $\mathrm{CDH12}$. Endothelial tube formation assay showed that down-regulation of $\mathrm{CDH} 12$ could obviously inhibit the process of angiogenesis, implying that $\mathrm{CDH} 12$ may play an important role in tumor metastasis.
\end{abstract}

Conclusion: Our results showed that $\mathrm{CDH} 12$ promotes proliferation, migration, invasion, adhesion and angiogenesis, suggesting that $\mathrm{CDH} 12$ may be an oncogene in colorectal cancer. $\mathrm{CDH} 12$ is expected to become a new diagnostic and prognostic marker and a novel target of the treatment of colorectal cancer.

Keywords: Cadherin12, Colorectal cancer, Cell proliferation, Cell migration, Cell invasion, Cell adhesion, Angiogenesis

\footnotetext{
*Correspondence: zhuzg@shsmu.edu.cn; adams_lu66@aliyun.com

${ }^{\dagger}$ Equal contributors

Shanghai Minimally Invasive Surgery Center, Shanghai Key Laboratory of Gastric Neoplasms, Department of Surgery, Shanghai Institute of Digestive Surgery, Ruijin Hospital, School of Medicine, Shanghai Jiao Tong University, 197 Rui Jin Er Rd, Shanghai 200025, People's Republic of China
} 


\section{Background}

Cadherin adhesion molecule is one of the ubiquitous types of cell-cell interactions required for the maintenance of complex tissue morphology. Traditionally, cadherins are membrane-spanning $\mathrm{Ca}^{2+}$-dependent homophilic adhesion receptors including E-cadherin, $\mathrm{N}$-cadherin, P-cadherin, VE-cadherin [1]. Classical cadherin molecules, especially Ecadherin and $\mathrm{N}$-cadherin are involved intimately in the pathogenesis of various cancers such as breast cancer, esophageal cancer, prostate cancer, gastric cancer etc. [2-5]. The loss of E-cadherin and the up-regulation of N-cadherin influence the behaviors of many types of cancers which process is also known as cadherin switch [6]. Considering the important function of cadherin in cell-cell contacts, they are usually postulated to play a vital role in cancer metastasis.

Colorectal cancer is one of the most common cancer types worldwide and its incidence increases with the change of life style and dietary structure [7]. Colorectal cancer has the propensity to colonize the liver and leads to a poor prognosis in the patient. However, metastasis is a complex process and has not been fully understood. We hypothesized that the destruction of cadherin adhesions acts as a promoter to facilitate the metastasis of colorectal cancer. In this article, we identified CDH12 as such an important molecule. CDH12 is also known as brain cadherin, because it is first identified in the brain and it can promote the differentiation of nervous system in mammary animal. In addition, CDH12 is associated with the pathogenesis of some neuropsychiatric disorders $[8,9]$. The comprehensive influence of classical cadherins on cancer such as E-cadherin and N-cadherin has been deeply clarified. However, the impact of CDH12 on cancer didn't attract more attention until recent years. Gemma Armengol et al. verified that CDH12 plays a significant role in the progression of non-small-cell lung cancer, and patients without CDH12 mutations have a longer survival than those with $\mathrm{CDH} 12$ mutation. This indicates that $\mathrm{CDH} 12$ acts as a tumor suppressor gene in the non-small-cell lung cancer [10]. In addition, CDH12 is able to promote the migration and invasion ability of salivary adenoid cystic carcinoma [11]. However, the function of CDH12 in colorectal cancer is unclear. In our research, we demonstrated the down-regulation of proliferation, invasion and migration ability in colorectal cancer cell lines by silencing CDH12 expression. Furthermore, we showed down-regulation of $\mathrm{CDH} 12$ can obviously inhibit the process of angiogenesis, implying that $\mathrm{CDH} 12$ may play an important role in tumor metastasis.

\section{Methods}

\section{Cell culture}

Seven human CRC cell lines were purchased from American Type Culture Collection (Manassas, VA,
United States). All the cell lines are preserved by Shanghai Digestive Surgery Institute. SW1116, SW480 and SW620 are cultured in Leibovitz's L-15 medium supplemented with $10 \%$ fetal calf serum, and $2 \mathrm{mM}$ L -glutamine. HCT116, HT29, Caco2, LoVo and NCM460 were maintained in RPMI-1640 medium with the same components. CRC cell lines were cultured in $37^{\circ} \mathrm{C}, 5 \% \mathrm{CO}_{2}$ incubator. Total RNA and protein were extracted after being incubated to $70-80 \%$ confluence.

\section{Tissue microarray and immunohistological analysis}

Tumor tissue and adjacent normal tissue of 56 patients with specific diagnosis of CRC and laparoscopic surgery in Minimally Invasive Surgery Centre, Ruijin Hospital, Shanghai Jiaotong University were collected from 2009 to 2011. The collection of specimens was authorized by Ethics Committee of Ruijin Hospital. None of the patients received preoperative treatment such as radiation or chemotherapy. The patients include 33 males, 23 females and the median age is 65.Staging of CRC tumor tissue was performed in accordance with the TNM classification provided by World Health Organization [12]. All the specimens fixed by $10 \%$ formaldehyde and embedded with paraffin were made into microarray. The staining of tissue microarray was conducted according to the manufacturer's protocol. After being dewaxed and hydrated, the microarray was antigen-retrieved by microwaving in citrate buffer $(10 \mathrm{mM}$ citric acid, $\mathrm{pH}$ 6.0 ), blocked in $5 \%$ animal serum, and stained for $2 \mathrm{~h}$ at $37^{\circ} \mathrm{C}$ by using primary antibody. Next ,the microarray was stained for $10 \mathrm{~min}$ at $37^{\circ} \mathrm{C}$ by using secondary biotinylated anti-rabbit antibodies at a 1:100 dilution followed by streptavidin-HRP. Specimens were developed by DAB and the nucleus was counterstained by Hematoxylin. The sections were photographed under a microscope and analyzed by two pathologists without knowing the pathological materials. Staining results were evaluated following the standard provided by Shimazui $\mathrm{T}$ et al. $[13,14]$. When the CDH12 were uniformly expressed, heterogeneously expressed or none-expressed in the tissue, the sections were evaluated as uniformly CDH12 positive $(+)$, heterogeneous $(+/-)$, or uniformly negative $(-)$, respectively.

\section{Quantitative reverse transcription polymerase chain reaction}

Total RNA was extracted with TRIzol (Invitrogen, Carlsbad, CA, United States) and the concentration was measured by spectrophotometer. Reverse transcription was performed to synthesize cDNA using reverse transcription kit(Invitrogen, CA, United States). The reaction was performed in an Applied Biosystems 7500 System with the mixture of cDNA, primers and Power SYBR Green PCR Master Mix (2×, Applied Biosystems, Warrington, 
United Kingdom).CDH12 PCR sense primer: 5'-AGGA GGTGGGGAGGAAGATA-3', antisense primer: 5'-CATATGTGGCCAGTGAATCG-3'. To quantify the expression level of CDH12, we use GAPDH as a control. GAPDH PCR sense primer: 5'-CCACGGAGCCGAAAACTAAAG3, antisense primer: 5'-GTAGCCCAGGATGCCCTTGA-3'.

\section{Western blot analysis}

Protein lysates were prepared from collected cells with RIPA and the supernatant was collected after being reacted for $30 \mathrm{~min}$ on the ice, centrifuged for $15 \mathrm{~min}$ at $13000 \mathrm{rpm}$. BCA (bicinchonininc acid) was used to determine protein concentration. An equal amount of protein $(50 \mu \mathrm{g})$ from each condition was subjected to $12.5 \%$ SDS-PAGE. Western blotting was carried out using the primary antibodies: rabbit polyclonal anti-CDH12 antibody (Abcam), 1:100; mouse polyclonal anti-GAPDH antibody,1:5000 for overnight at $4^{\circ} \mathrm{C}$, followed by incubation with fluorescence-labeled secondary antibody for 2 $\mathrm{h}$ at room temperature. LI-COR Odyssey Infrared fluorescence scanner was used to capture the images.

\section{RNAi}

$3 \times 10^{5}$ cells were seeded into six-well plate each chamber. Until 70\% confluence, GFP-labeled shRNA targeting CDH12 was transfected into cells mediated by Lipofectamine ${ }^{\mathrm{TM}} 2000$ according to the instruction. Cell sections transfected with GFP-labeled shRNA targeting nonhomologous gene to $\mathrm{CDH} 12$ and Lipofectamine ${ }^{\mathrm{TM}} 2000$ only were performed as control. After 12 hours transfection, cells were observed under fluorescence microscope.

\section{ShRNA oligonucleotides screen}

The following shRNA oligonucleotides were used to interfere $\mathrm{CDH}-12$ expression and the most effective oligonucleotide was screened with determining the mRNA level using quantitative PCR:shCDH12-1: 5'-CACCGCT GGGCAACAATTCTCCTTT

\section{TCAAGAGAAGGAGAATTGTTGC CCAGC \\ TTTTTG-3'(sense sequence), shCDH12\#2:5' - CACCGCGCAGTATAATTTCTCCA TAC TCGAGTATGGAGA AATTATACTGCGCTTTTTG-3'(sense sequence), shCDH12\#3:CACCCGGTCACATTTCCAACGTGT TCTCGAGAACACGTTGGAAATGTGACCG TTTTTG-3'(sense sequence), Negative control: 5'-CACCGT TCTCCGAACGTG TCACGTCAAGAG ATTACGTG ACACGTTCGGAG AATTTTTTG-3'(sense sequence).}

\section{Cell viability assay}

Cells were seeded in 96-well plate and cultured in $37^{\circ} \mathrm{C}$, $5 \% \mathrm{CO}_{2}$ incubator. Three groups (shCDH12 group, shNC group and Mock group) were designed with 6 copies each group. The viability of cells was determined at $0 \mathrm{~h}, 24 \mathrm{~h}, 48 \mathrm{~h}, 72 \mathrm{~h}, 96 \mathrm{~h}$ and $120 \mathrm{~h}$. At the test point, $10 \mu \mathrm{L}$ CCK- 8 was added into each well and the plate was incubated at $37^{\circ} \mathrm{C}$ for $2 \mathrm{~h}$ followed $\mathrm{OD}$ detection using spectrophotometer.

\section{Cell invasion and migration assay}

Transwell chamber was used $(8 \mathrm{~mm}, 24$-well format; Corning, Lowell, MA, USA) to perform cell invasion and
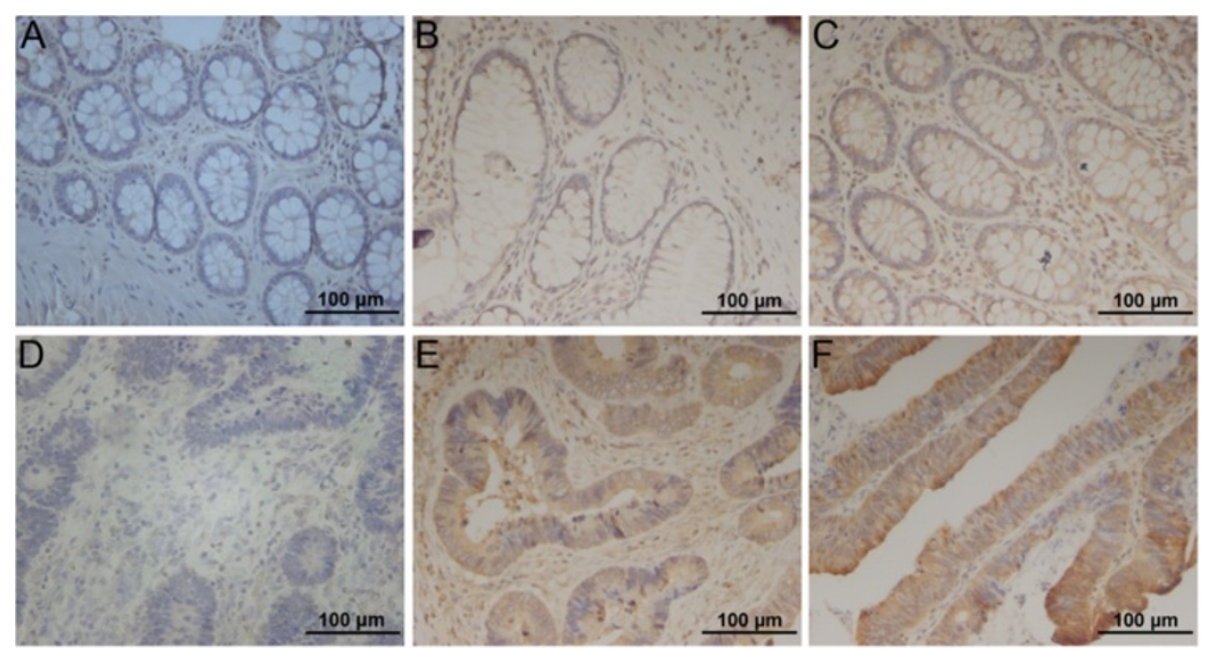

Figure 1 Immunohistochemical results of $\mathrm{CDH} 12$ expression in surgical specimens of tumor tissues or adjacent normal tissue from patients with colon cancer. A-C: Uniformly negative $(-)$, heterogeneous (+/-), and uniformly positive (+) staining in adjacent normal mucosa (200x); D-F: Uniformly negative (-), heterogeneous (+/-), and uniformly positive (+) staining in CRC tumor tissues (200x). 
Table 1 The expression of CDH12 in tumor tissues and adjacent normal tissues

\begin{tabular}{llllll}
\hline Classification & $\mathbf{n}$ & $\mathbf{C D H 1 2 ( - )}$ & $\mathbf{C D H 1 2 ( + / - )}$ & $\mathbf{C D H 1 2 ( + )}$ & $\boldsymbol{P}$ value \\
\hline Tumor tissue & 56 & 5 & & 41 & $<0.001$ \\
Normal tissue & 56 & 23 & 24 & 9 & \\
\hline
\end{tabular}

migration assay. In migration assay, $200 \mu \mathrm{L}$ Leibovitz's L-15 serum-free medium containing $3 \times 10^{5}$ cells was added into the upper chamber and $600 \mu \mathrm{L}$ Leibovitz's L15 with $10 \%$ serum was added into the lower chamber. The chamber was cultured in $37^{\circ} \mathrm{C}, 5 \% \mathrm{CO}_{2}$ condition for $24 \mathrm{~h}$. In invasion assay, the insert membranes were coated with diluted Matrigel (BD Biosciences) and performed in the same way as migration assay. Finally, cells on the top side of the inserts were removed with cotton swab. Chambers were fixed with methanol and Cells under the inserts were stained with $1 \%$ crystal violet for $30 \mathrm{~min}$ before rinsing with phosphate buffered saline (PBS) for $20 \mathrm{~min}$. The results are shown as means \pm sd. Each experiment was done in triplicate. The wound healing assays was performed as previously described $[15,16]$.

\section{Flow cytometric cycle analysis}

CRC cells were collected after $24 \mathrm{~h}, 48 \mathrm{~h}$ respectively and rinsed by PBS for twice. Collected cells were fixed in $75 \%$ iced ethanol for detection. Fixed cells was recollected by washing in PBS for twice, centrifuging at 1800 rpm for $5 \mathrm{~min}$. RNAase $(3 \mu \mathrm{L})$ and Propidium iodide $(\mathrm{PI}, 50 \mu \mathrm{L}$ ) were added into each tube, incubated for 30 min at $37^{\circ} \mathrm{C}$ in the darkness. The Cell cycle analysis was performed using BD FACS Vantage System (Becton, Dickinson and Company, Franklin Lakes, NJ, United States) according to the manufacturer's protocol.

\section{Cell aggregation assay}

Cells were harvested with trypsin/EDTA and resupended at $1.5 \times 10^{5}$ cells per ml in RPMI-1640 medium with FBS. $20 \mu \mathrm{L}$ drops containing 5000 cells per drop were pipetted onto the inner surface of the lid of petri plate. The lid were then reversed and placed on the petri plate with the drop hanging on the lid so that the cells failed to adhere to the petri plate. $4 \mathrm{ml}$ of PBS was placed in the petri plate to eliminate evaporation within the hanging drops and the results were observed under microscope.

\section{Cell adhesion assay}

Human umbilical vein endothelial cells (HUVEC) were plated into 24-well plate and incubated at $37^{\circ} \mathrm{C}, 5 \% \mathrm{CO}_{2}$ for $24 \mathrm{~h}$ to form monolayer. Tumor cells after being managed respectively were collected with trypsin/EDTA and resuspended by RPMI-1640 medium. $700 \mu \mathrm{L}$ medium containing 20,000 cells were subsequently added into each well and incubated in the incubator for $6 \mathrm{~h}$. Subsequently, medium in each well was abandoned and the wells were washed by PBS for three times. Results were observed under fluorescence microscope and five random views were chosen to quantify.

\section{Endothelial tube formation assay}

Each well of prechilled 96-well plates was coated with a thin layer of the Matrigel $(50 \mu \mathrm{L} /$ well $)$ which was incubated to polymerize at $37^{\circ} \mathrm{C}$ for $1 \mathrm{~h}$. Human umbilical vein endothelial cells (HUVEC) were resuspended in the supernants collected from shRNA group, control groups. Add $300 \mu \mathrm{L}$ of the supernants to each well containing $4 \times 10^{4}$ HUVEC cells and incubated at $37^{\circ} \mathrm{C}, 5 \% \mathrm{CO}^{2}$ for

Table 2 Relationship between CDH12 expression level and clinicopathologic variables in 56 CRC patients

\begin{tabular}{lllll}
\hline Classification & CDH-12(-) & CDH-12(+/-) & CDH-12(+) & P value \\
\hline Gender & & & & \\
Male & 3 & 7 & 23 & 0.725 \\
Female & 2 & 3 & 18 & \\
Age (year) & & & & \\
$\leq 65$ & 2 & 4 & 19 & 0.915 \\
$>65$ & 3 & 6 & 22 & \\
Tumor location & & & & \\
right hemicolon & 0 & 1 & 11 & 0.283 \\
left hemicolon & 1 & 1 & 1 & \\
sigmoid colon & 2 & 4 & 8 & \\
rectum & 2 & 4 & 21 & \\
Tumor size (cm) & & & & \\
$\leq 4 \times 3$ & 3 & 7 & 23 & 0.725 \\
$>4 \times 3$ & 2 & 3 & 18 & \\
TNM stage & & & & \\
invasion depth & & & & \\
$\mathrm{T}_{1}$ & 2 & 0 & 1 & 0.024 \\
$\mathrm{~T}_{2}$ & 1 & 1 & 4 & \\
$\mathrm{~T}_{3}$ & 2 & 8 & 34 & \\
$\mathrm{~T}_{4}$ & 0 & 1 & 2 & \\
lymph node & & & & \\
$\mathrm{N}_{0}$ & 1 & 5 & 18 & 0.237 \\
$\mathrm{~N}_{1}$ & 4 & 2 & 16 & \\
$\mathrm{~N}_{2}$ & 0 & 3 & 7 & \\
organ metastasis & & & & \\
$\mathrm{M}_{0}$ & 4 & 10 & 38 & \\
$\mathrm{M}_{1}$ & 1 & 0 & 3 & \\
\hline
\end{tabular}



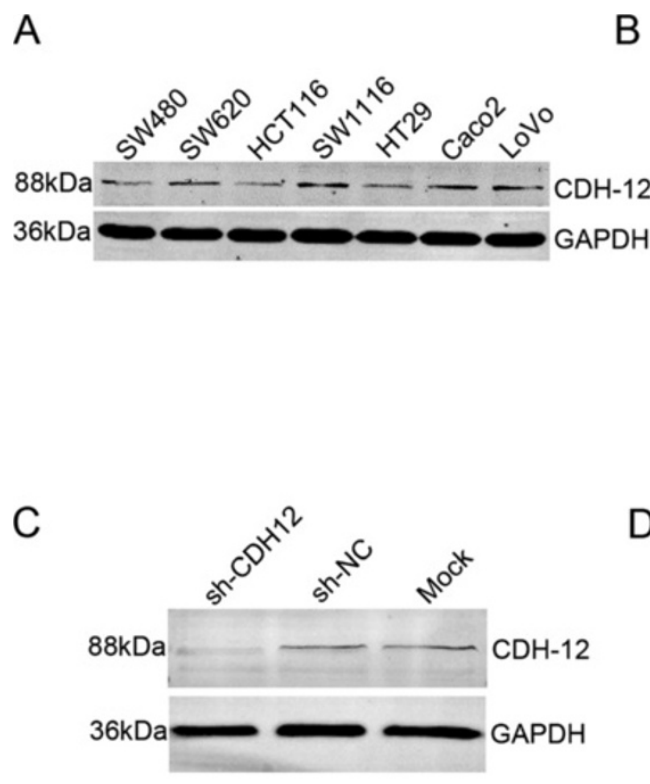

$\mathrm{B}$

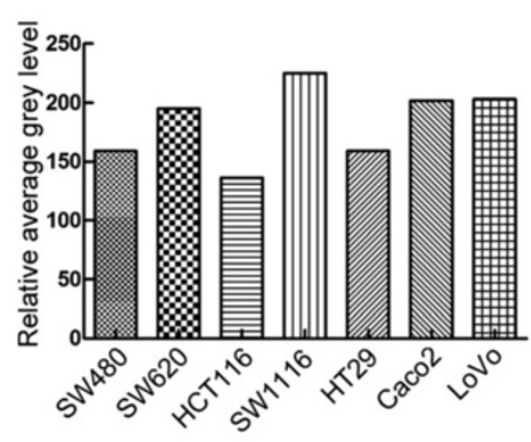

D

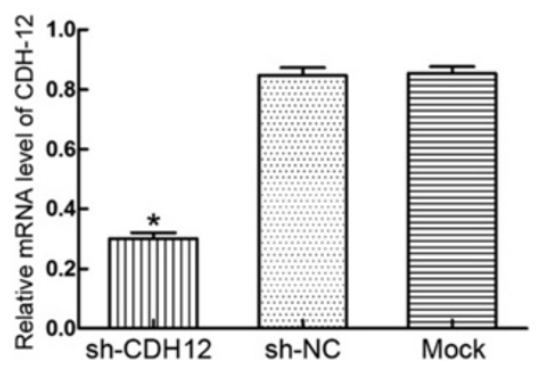

Figure 2 Expression of $\mathrm{CDH} 12$ in CRC cell lines and the interfering effect of shRNA. A: CDH12 expression in CRC cell lines detected by western blot; $\mathbf{B}$ : Relative average grey level of western blot bands; $\mathbf{C}$ : Interfering effects of shCDH12 in SW1116 were detected using western blot; D: mRNA expression levels of CHD12 in SW1116 cell lines transfected with shRNAs $\left({ }^{*} P<0.05\right)$.

24 h. Five random views were choosed to evaluate tube formation ability by counting the tubular number, the tubular length and tubular intersecting nods in using Image Pro Plus software (Media Cybernetics Inc., Bethesda, MD, USA) according to Mirshahi's method [17]. Each experiment was performed at least three times.

\section{Statistical analysis}

All the data were analyzed by SAS 8.0 statistical software. The difference of CDH12 expression between tumor tissue and adjacent normal tissue was examined by Cochran- Maantel-Terpstra test. Chi-square test and Fisher exact probability method was used to analyze the relationship between $\mathrm{CDH} 12$ expression level and clinical features. Survival probabilities were calculated using Kaplan-Meier method, differences between two groups were determined using the log-rank test. Multiple comparisons were performed by one-way

Table 3 The absorbance in the proliferation assay

\begin{tabular}{llllll}
\hline Group & $\mathbf{2 4} \mathbf{h}$ & $\mathbf{4 8} \mathbf{h}$ & $\mathbf{7 2} \mathbf{h}$ & $\mathbf{9 6} \mathbf{h}$ & $\mathbf{1 2 0} \mathbf{h}$ \\
\hline shCDH12 & $0.28 \pm 0.038$ & $0.49 \pm 0.060$ & $0.62 \pm 0.078$ & $1.08 \pm 0.245$ & $2.68 \pm 0.531$ \\
shNC & $0.33 \pm 0.023$ & $0.62 \pm 0.108$ & $1.06 \pm 0.121$ & $3.02 \pm 0.168$ & $3.82 \pm 0.125$ \\
Mock & $0.38 \pm 0.105$ & $0.63 \pm 0.095$ & $1.36 \pm 0.131$ & $3.23 \pm 0.125$ & $3.92 \pm 0.043$ \\
\hline
\end{tabular}

analysis of variance. $P<0.05$ was considered statistically significant.

\section{Results}

The expression levels of $\mathrm{CDH} 12$ in CRC patients are significantly correlated with invasion depth.

Immunohistochemistry tissue microarray staining was performed to detect the expression of $\mathrm{CDH} 12$ in the $\mathrm{CRC}$ tissue, and the representative results were shown in Figure 1. 41 cases out of the 56 tumor samples exhibited a substantial increase in overall CDH12 expression, 10 cases expressed heterogeneously and 5 cases expressed uniformly negative. Obvious difference between tumor tissues and adjacent normal tissues was verified by statistical analysis, the positive rate is significantly higher in the tumor tissues compared to the adjacent normal tissues (Table 1). The expression level of CDH12 is significantly correlated with invasion depth $(P=0.024)$. However, there are no significant relationships between the CDH12 expression and other clinicopathologic features such as gender, age, tumor size, or lymph node metastasis $(P>0.05)$ (Table 2). In addition, to further elucidate the clinico-pathologic significance of CDH12 overexpression in CRC with prognosis, we correlate CDH12 expression in CRC tissues with the survival time 44 out of 50 patients that were successfully followed up. 

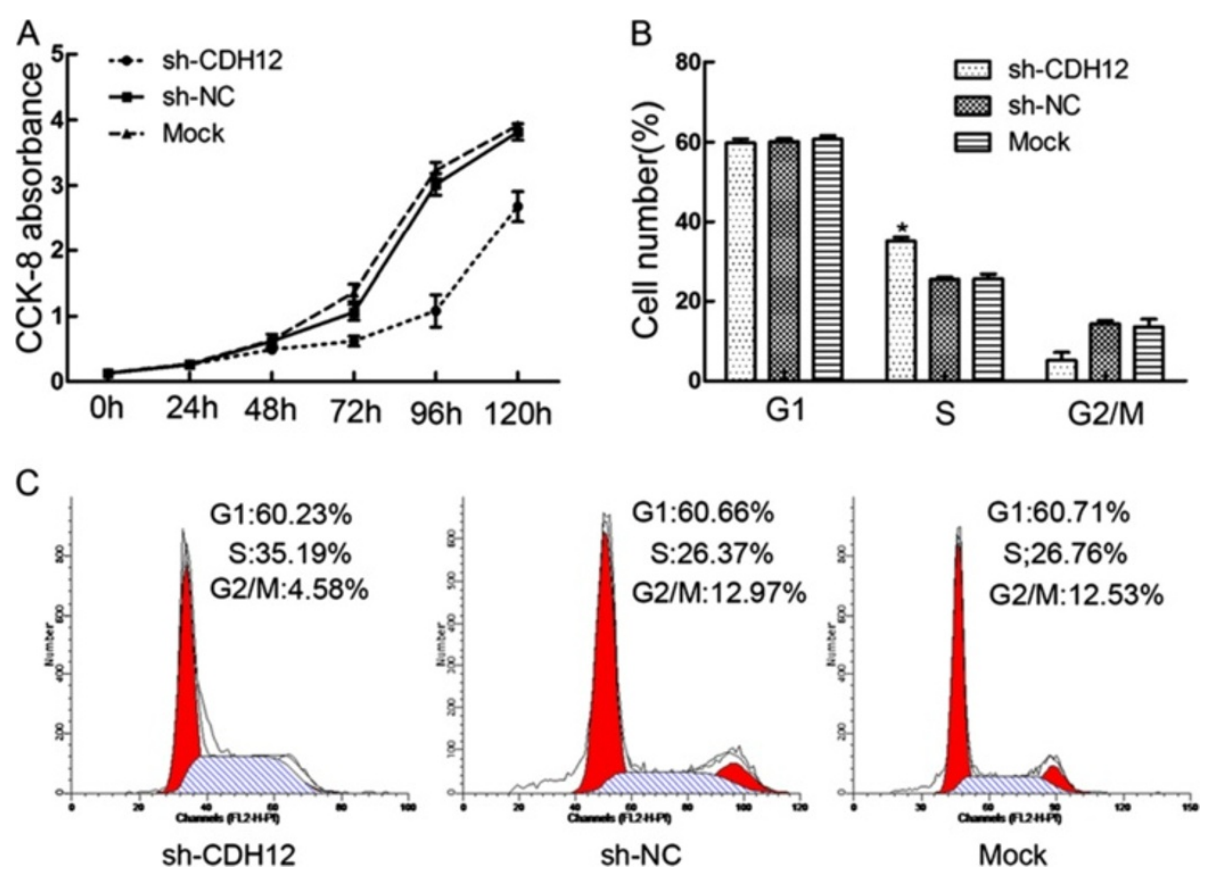

Figure 3 Effect of CDH12 on cell proliferation and cell cycle in CRC cell lines. A. Cell numbers at 0,24, 48, 72,96 and $120 \mathrm{~h}$ was measured by CCK-8 assays. B: Proportion of cells in various phases of the cell cycle. The results are means of three independent experiments \pm S.D $(*: P<0.05)$. C: Representative histograms depicting cell cycle profiles of cells transiently transfected with shCDH12 or controls.

The Kaplan-Meier survival analysis showed the survival rate of the CDH12 positive patients group was significantly lower than the CDH12 negative patients group $(P<0.05$, Additional file 1: Figure S1).

\section{Expression of $\mathrm{CDH} 12$ in $\mathrm{CRC}$ cell lines and the interfering effect of shRNA}

Western blot was used to detect the CDH12 expression in CRC cell lines, including the non-cancer cell line NCM460. CDH12 was expressed in all these cell lines, and especially high in SW1116 and SW620, but low in HT29 and HCT116 (Figure 2B\&C, Additional file 2: Figure $\mathrm{S} 2 \mathrm{~B} \& \mathrm{C}$ ). In order to explore the functions of CDH12 in CRC cell lines, we chose the CHD12 highexpression cell lines SW1116 and SW620. The three shRNAs targeting CDH12 or a scramble control shRNA was transfected into SW1116 or SW620 and the expressions of CDH12 were detected (Additional file 2: Figure $\mathrm{S} 2 \mathrm{~A})$. The results showed that the expression of CDH12 was significantly decreased in shRNA-CDH12 transfection cells compared with the control groups (Figure 2C, 2D).

\section{Down-regulation of $\mathrm{CDH} 12$ can inhibit $\mathrm{CRC}$ cells proliferation and block cell cycle progression}

We next determined whether CDH12 could affect the cell proliferation and cell cycle. The proliferation ability of SW1116 was examined at six time points $(0 \mathrm{~h}, 24 \mathrm{~h}, 48 \mathrm{~h}$, $72 \mathrm{~h}, 96 \mathrm{~h}$ and $120 \mathrm{~h}$ ) after being transfected with shRNAs. The results was shown as means \pm SD (Table 3 ) and the proliferation curves (Figure 3A). Cell proliferation ability in shCDH12 group was decreased significantly compared with the control groups $(P<0.01)$. Consistently, ectopic expression of CDH12 in HT29 and HCT116 promotes the proliferation of the CRC cells (Additional file 3: Figure S3A\&D, $P<0.01)$.

To further detect the influence of CDH12 on cell cycle, we performed flow cytometric cell cycle analysis to examine which phase of cell cycle was influenced by CDH12 in SW1116. Flow cytometric analysis demonstrated that proportions of cells in shCDH12 group at $\mathrm{S}$ phase is more than that in shNC group and Mock group $\left({ }^{*} P<0.05\right.$, Figure $3 \mathrm{~B} \& \mathrm{C})$. These data indicated that down-regulation of CDH12 could obviously block cells in S phase.

\section{Down-regulation of $\mathrm{CDH} 12$ inhibits wound healing ability,} migration and invasion of CRC cells

Wound healing assay was used to examine migration ability of SW1116 and SW620. Cells was plated in 12well plate after being transfected and scratched by $20 \mu \mathrm{L}$ sterile pipet tips. Results were showed in Figure 4A\&C. Three lines were drawn in each group and relative length were calculated which is showed in Figure 4B\&D. After $48 \mathrm{~h}$ incubation, the distance of the scratch woud in shCDH12 group is significantly larger compared with control groups. (The woud diatance: shCDH12 group: $1.07 \pm 0.11$, shNC group: $0.17 \pm 0.06$, Mock group: $0.20 \pm 0.17$ ), The similar results were observed in theSW620 cell line (shCDH12 group: $1.08 \pm 0.05$, shNC group: $0.18 \pm 0.06$, Mock 

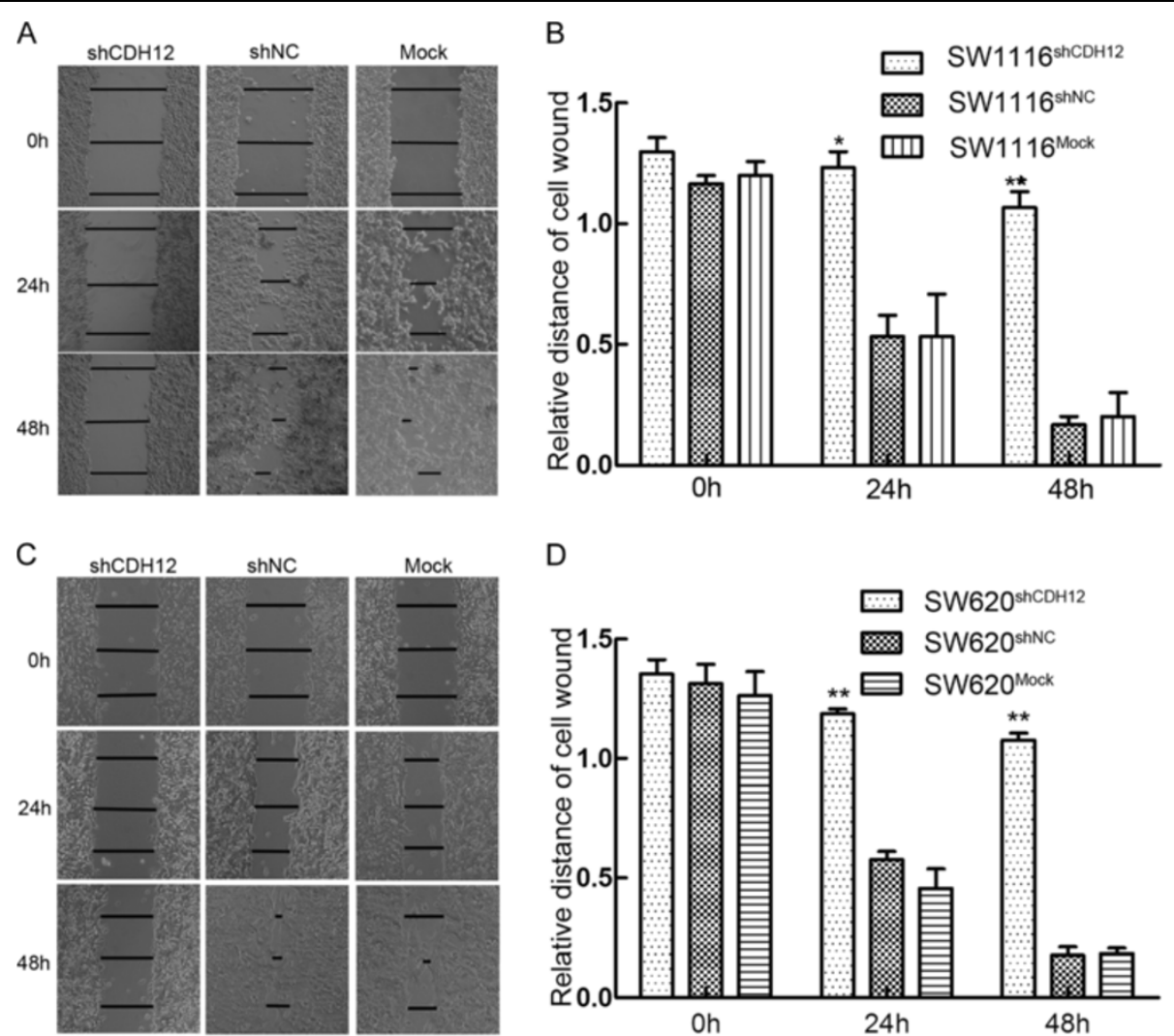

Figure 4 Wound healing assay. A: Representative photographs of scratch wounds in SW1116 (100X), B: Relative distances of cell wounds in shCDH12, shNC and Mock group (*:P<0.05), C: Representative photographs of scratch wounds in SW620 (100x); B: Relative distances of cell wounds in shCDH12, shNC and Mock group (**:P<0.01).

group: $0.18 \pm 0.04)$. Consistently, ectopic expression of CDH12 in HT29 and HCT116 promotes wound healing ability of CRC cells. (HT29, CDH12 group: 0.18 \pm 0.05 , Vector group: $0.99 \pm 0.13$; HCT116, CDH12 group: 0.20 \pm 0.06 , Vector group: $0.83 \pm 0.07$,) (Additional file 3: Figure S3 B\&C and $\mathrm{E} \& \mathrm{~F})$.

To further detect the influence of CDH12 on migration and invasion, we performed transwell migration and invasion assay. In transwell migration assay, the number of cells migrating through the chamber in shCDH12 was $85 \pm 18.42$ which was lower than in shNC

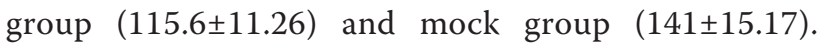
The same result was also observed in the invasion assay: shCDH12 group: $37.80 \pm 11.28$, shNC group: $78.40 \pm 13.05$, Mock group: 93.60 \pm 8.91 (Figure 5A-D, $\left.{ }^{*} P<0.05\right)$. Similarly, the results are implemented in SW620 cell lines. (Migration assay: shCDH12 group: $78.4 \pm 10.38$, shNC group: $120 \pm 8.7$, Mock group: $135 \pm 9.8$. sh; Invasion assay: shCDH12 group: $33 \pm 10.07$, sh-NC group: 55.8 \pm 9.52 , Mock group: $60.8 \pm 10.87)$. (** $P<0.05$, Figure 5E-H). Consistently, ectopic expression of CDH12 in HT29 and HCT116 promotes the migration and invasion of CRC cells (Additional file 4: Figure $S 4,{ }^{*} P<0.05$, ** $P<0.01)$.

\section{CDH12 can promote cell-cell junction formation}

As a member of the cadherin family, CDH12 is likely to influence the cell-cell junction. We firstly performed the cell aggregation assay to examine the role of CDH12 in cell-cell junction. The results demonstrated that suppression of CDH12 can inhibit the formation of cell colony because the cells in $\mathrm{CDH} 12$ knock-down groups distribute more dispersedly compared with control groups (Figure 6A). We performed adhesion assay subsequently to examine the adhesion ability of SW1116 to endothelial cells after CDH12 being decreased. After being washed with PBS, the number of SW1116 cells adhered to the HUVEC monolayer in the shCDH12 group was less than the shNC group (Figure 6B, 6C).These suggest that $\mathrm{CDH} 12$ is an essential adhesion molecule not only to tumor cells junctions but also to the adhesion between tumor cells and endothelial cells.

\section{CDH12 can promote endothelial tube formation}

Human umbilical vein endothelial cells (HUVECs) $\left(4 \times 10^{4}\right.$ cells/well $)$ were suspended in supernatants collected from shCDH12 group, shNC group and Mock group. After being incubated for $24 \mathrm{~h}$, tubular numbers of each group were assessed under microscope. The 


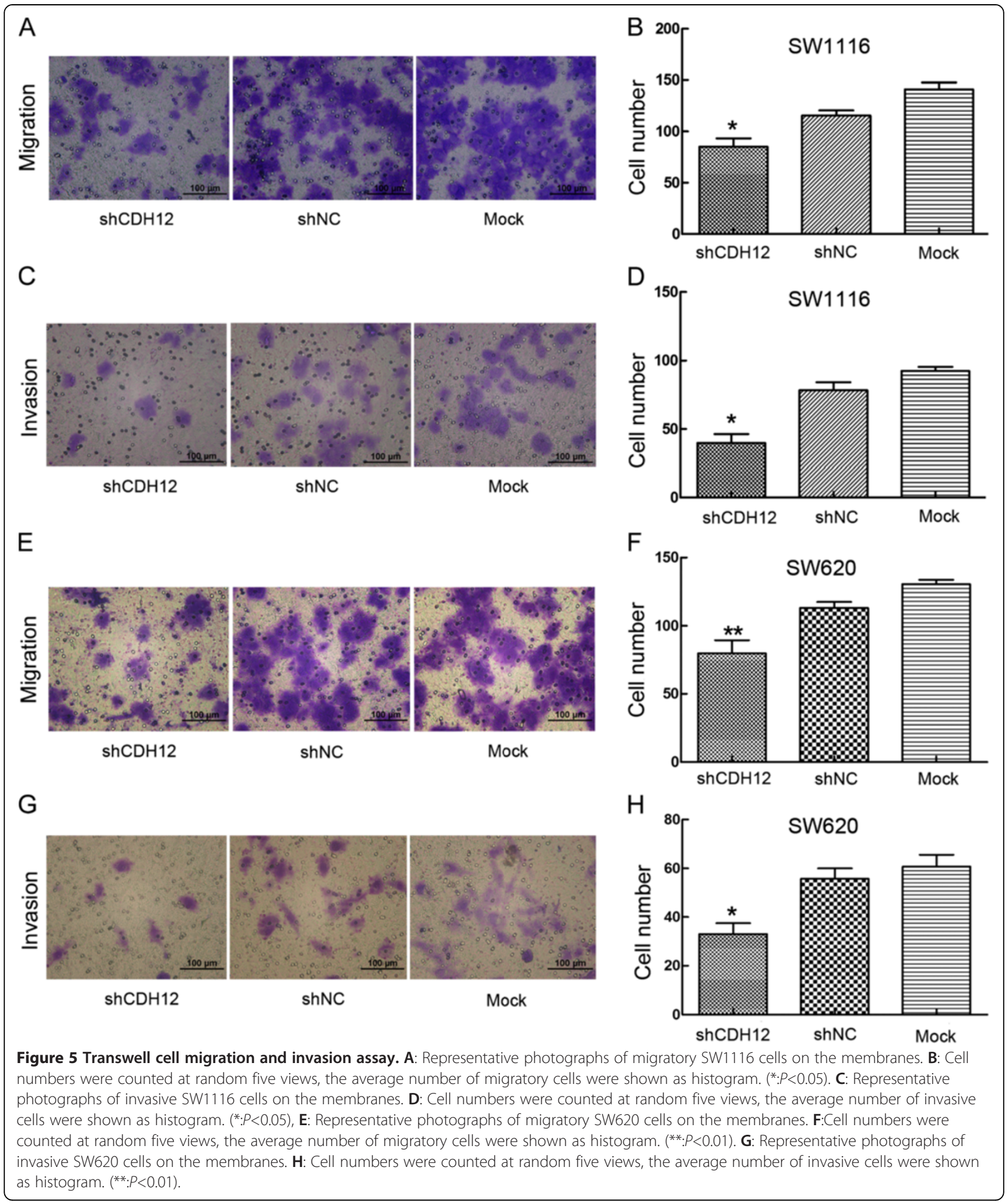

supernatant from shCDH12 group presented strong inhibiting effect on endothelial tube formation compared with shNC group and Mock group whatever in tubular number and tubular intersecting nods (Figure 7).

\section{Discussion}

CDH12 protein belongs to type II transmembrane protein containing 794 amino acids with a molecule weight of $88 \mathrm{kDa}$ and functions in the $\mathrm{Ca}^{2+}$-dependent cell-cell 
A

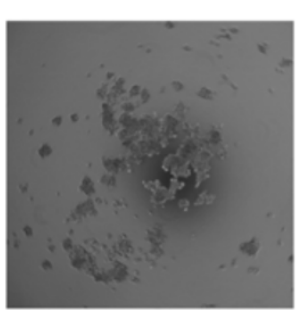

sh-CDH12

B

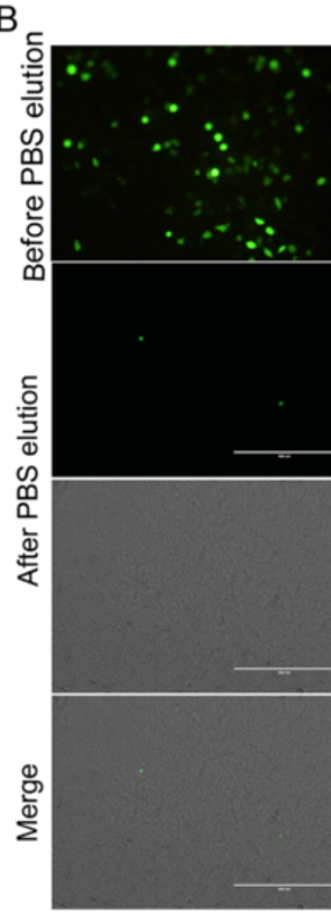

sh-CDH12

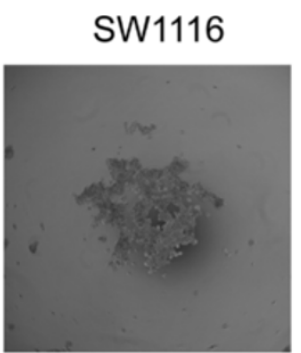

sh-NC

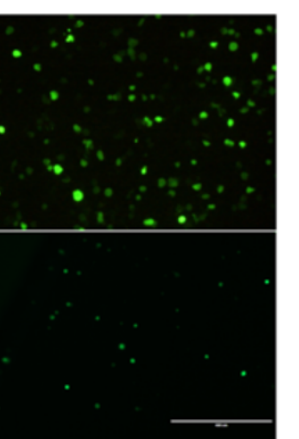

C

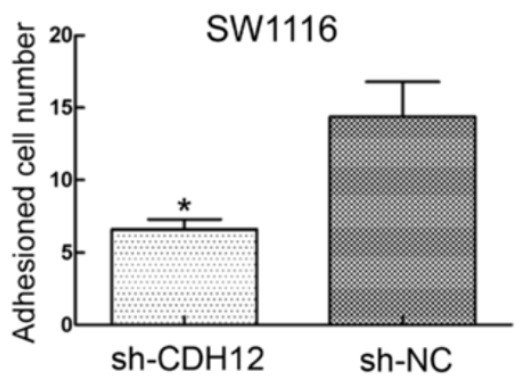

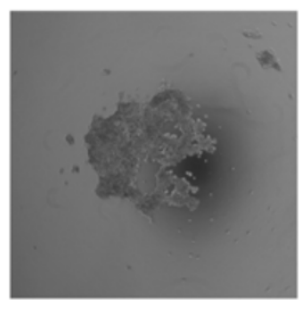

Mock

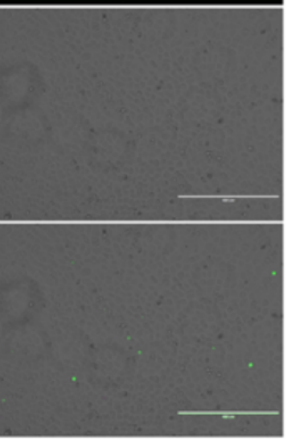

sh-NC

Figure 6 Cell aggregation assay and cell adhesion assay. A:The formation of cell colony was obviously inhibited in shCDH12 group compared with control groups. B: Cell numbers adhered to HUVEC monolayer in shCDH12 group is less than negative control group after PBS elution which demonstrated the function of $\mathrm{CDH} 12$ in tumor cell and endothelial cell junction. As the transfectant (shRNA and scrambled shRNA) is marked with GFP, so the cells adhered to the monolayer was showed as green dots. C: The numbers of the cells were counted in five random views and the statistical result is presented as histogram $\left({ }^{*} P<0.05\right)$.

junction. CDH12 can promote the migration and invasion ability of salivary adenoid cystic carcinoma [11]. According to our findings, CDH12 may be an oncogene and promote the malignant progression of CRC cells.

Our findings showed that CDH12 expression is substantially upregulated in CRC tissues compared to adjacent normal tissue according to the histochemistry microarray analysis, indicating that CDH12 may also contribute to tumorigenicity in CRC. In addition, the expression of CDH12 is significantly associated with invasion depth. These data suggests that CDH12 may be associated with CRC invasion and metastasis. We selected the CDH12 high-expression cell lines SW1116 and SW620, and successfully inhibited CDH12 expression in them by shRNA. After CDH12 abrogation, the migration and invasion ability of SW1116 or SW620 was severely impaired.

These results verified the important role of CDH12 in CRC cells migration and invasion behavior and which may be explained that CDH12 is related to several intracellular metastasis-associated signal molecules that can promote tumor cell migration. This is consistent with the report that $\mathrm{CDH} 12$ is able to promote the migration and invasion ability of salivary adenoid cystic carcinoma [11].

We also performed cell aggregation assay and adhesion assay to further determine the importance of CDH12 in intercellular junction. Cell aggregation assay showed that the absence of CDH12 leads cells to distribute more dispersedly compared with control groups. 


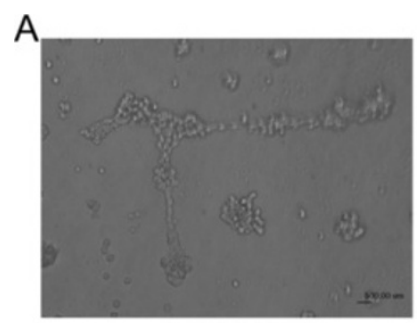

sh-CDH12

B

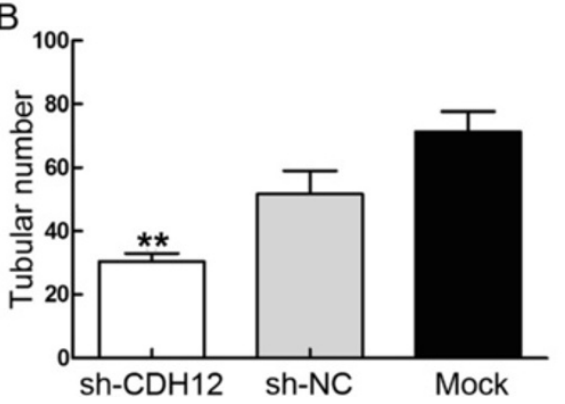

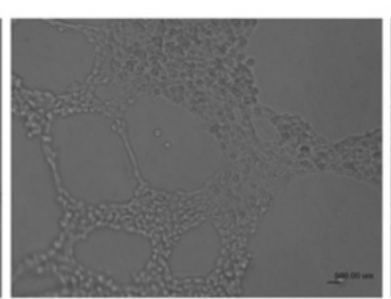

sh-NC

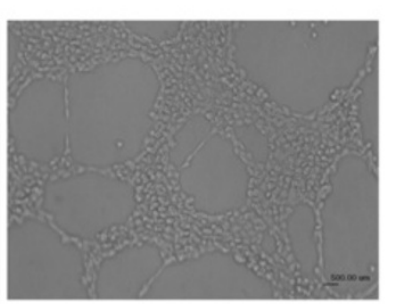

Mock

C

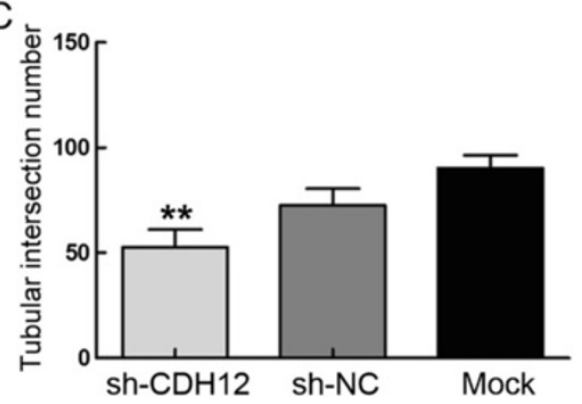

Figure 7 Endothelial tube formation assay. Effect of suppressing $\mathrm{CDH} 12$ expression on tubular formation in vitro. The supernatants from cells with or without $\mathrm{CDH} 12$ suppression were collected which was used to suspend HUVEC. After $24 \mathrm{~h}$ co-cultivation, tubular formation was evaluated. A: Poorly formed tubular structure was observed in shRNA group, compared with negative control group and mock group. B: The chart represent the counting of tubules numbers ( $\left.{ }^{* *} P<0.001\right)$; $\mathbf{C}$ : The intersecting nods ( $\left.{ }^{* *} P<0.001\right)$, The data represent the mean \pm SD of three independent experiments.

Destruction of cell-cell junction is an initial reaction of tumor cell metastasis which manifests with repression of cell adhesion molecules. This process may be also known as epithelial-mesenchymal transition (EMT) [18]. Reduced intracellular adhesion may allow tumor cells to disseminate and spread throughout the extracellular matrix. Generally, tumor cells gaining the ability to leave the site and metastasize are accompanied with "cadherin switching" [6]. CDH12 may also promote tumor metastasis through this switching mechanism. Simultaneously, adhesion assay that cells numbers attached to the HUVEC monolayer in shCDH12 group were less than the control group, revealed the importance of CDH12 in the adhesion of tumor cells to endothelial cell. In the process of tumor metastasis, the arrest of circulating tumor cells to luminal walls of distant organ microvasculature is an essential part of this process [19]. Collectively, the result demonstrates that $\mathrm{CDH} 12$ may promote tumor cells to attach to the vascular enthelial cells during tumor metastasis.

Tumors require supplements of nutrients and oxygen and evacuation of metabolic wastes and carbon dioxide as normal tissues. The tumor-associated neovasculature, generated by the process of angiogenesis, not only supplies abundant nutrients for tumor cell but also provides conveniences for tumor cell dissemination [20]. Our results showed that down-regulation of $\mathrm{CDH} 12$ on SW1116 or SW620 cells effectively inhibited the formation of vessel. The numbers of tube, tubular length and tubular intersecting nods in shRNA group is less than the control. This suggests that $\mathrm{CDH} 12$ can promote the process of angiogenesis. Considering the importance of angiogenesis in tumor metastasis and the contribution of $\mathrm{CDH} 12$ in tumor cell migration, invasion and cell cycle, CDH12 maybe a potential target to supervise tumor cell malignant metastasis.

\section{Conclusion}

Our results showed that $\mathrm{CDH} 12$ promotes proliferation, migration, invasion, adhesion and angiogenesis, suggesting that $\mathrm{CDH} 12$ may be an oncogene in colorectal cancer. $\mathrm{CDH} 12$ is expected to become a new diagnostic and prognostic marker and a novel target of the treatment of colorectal cancer. More efforts should be put to clarify the signal pathways underlying these biological phenomenon.

\section{Additional files}

Additional file 1: Figure S1. Kaplan-Meier survival analysis of $\mathrm{CDH} 12$ expression in 50 CRC patients. The Kaplan-Meier survival analysis showed the survival rate of the $\mathrm{CDH} 12$ positive patients group was significantly lower than in the $\mathrm{CDH} 12$ negative patients group.

Additional file 2: Figure S2. Expression of $\mathrm{CDH} 12$ in $\mathrm{CRC}$ cell lines and the result of shRNAs sequence screening. A: The Interfering effects of three shRNAs targeting CDH12 in SW1116 and SW620, B: CDH12 expression in CRC cell lines detected by western blot; C: Relative average grey level of western blot bands.

Additional file 3: Figure S3. Effect of enforcing $\mathrm{CDH} 12$ on cell proliferation and wound healing in CRC cell lines. A: Growth curves of 
HT29 cell lines were measured by CCK-8 assays. B: Representative photographs of scratch wounds in HT29 (100x), C: Relative distances of cell wounds in CHD12 ectopic expression group and control group ${ }^{*} P<0.05$, $\left.{ }^{*} P<0.01\right)$, D: Growth curves of HT29 cell lines were measured by CCK-8 assays. E: Representative photographs of scratch wounds in HCT116 (100x); F: Relative distances of cell CHD12 ectopic expression group and control group $\left({ }^{*} P<0.05,{ }^{* *} P<0.01\right)$

Additional file 4: Figure S4. Ectopic expression of $\mathrm{CDH} 12$ promotes migration and invasion of CRC cells. A: Representative photographs of migratory or invasive HT29 cells on the membrane. B: Average number of migratory or invasive HT29 cells $\left({ }^{*} P<0.05\right)$. C: Representative photographs of migratory or invasive HCT116 cells on the membrane. D: Average number of migratory or invasive HCT116 cells $\left({ }^{*} P<0.05\right)$. The data represent the mean \pm s.d. of three independent experiments.

\section{Abbreviations}

CDH12: Cadherin 12; CRC: Colorectal carcinoma; EMT: Epithelialmesenchymal transition; BCA: Bicinchonininc acid; HUVECs: Human umbilical vein endothelial cells.

\section{Competing interests}

The authors declare that they have no competing interests.

\section{Authors' contributions}

AGL, ZGZ, MHZ and CXH conceived the study design, participated in its design and in the acquisition of data. JKZ and PL carried out the experiments, participated in the acquisition of data, analysis and interpretation, drafted the manuscript. HF, XZPW, YPZ and JJM has been involved in analyzing the data and drafting the manuscript. ZZ helped to draft and revise the manuscript. All authors read and approved the final manuscript.

\section{Acknowledgements}

This study was supported by Shanghai National Science Foundation (124119a0900) and National Natural Science Foundation of China (81300290, 81201625 and 81372187).

Received: 20 May 2013 Accepted: 21 October 2013

Published: 15 November 2013

\section{References}

1. Gumbiner BM: Cell adhesion: the molecular basis of tissue architecture and morphogenesis. Cell 1996, 84:345-357.

2. Li K, He W, Lin N, Wang X, Fan QX: Downregulation of N-cadherin expression inhibits invasiveness, arrests cell cycle and induces cell apoptosis in esophageal squamous cell carcinoma. Cancer Invest 2010, 28:479-486.

3. Yang Z, Zhang X, Gang H, Li X, Li Z, Wang T, Han J, Luo T, Wen F, Wu X: Up-regulation of gastric cancer cell invasion by Twist is accompanied by $\mathrm{N}$-cadherin and fibronectin expression. Biochem Biophys Res Commun 2007, 358:925-930.

4. Hulit J, Suyama K, Chung S, Keren R, Agiostratidou G, Shan W, Dong X, Williams TM, Lisanti MP, Knudsen K, Hazan RB: N-cadherin signaling potentiates mammary tumor metastasis via enhanced extracellular signal-regulated kinase activation. Cancer Res 2007, 67:3106-3116.

5. Alexander NR, Tran NL, Rekapally H, Summers CE, Glackin C, Heimark RL: $\mathrm{N}$-cadherin gene expression in prostate carcinoma is modulated by integrin-dependent nuclear translocation of Twist1. Cancer Res 2006, 66:3365-3369.

6. Wheelock MJ, Shintani Y, Maeda M, Fukumoto Y, Johnson KR: Cadherin switching. J Cell Sci 2008, 121:727-735.

7. Center MM, Jemal A, Ward E: International trends in colorectal cancer incidence rates. Cancer Epidemiol Biomarkers Prev 2009, 18:1688-1694.

8. Redies C, Hertel N, Hubner CA: Cadherins and neuropsychiatric disorders. Brain Res 2012, 1470:130-144.

9. Mayer M, Bercsenyi K, Geczi K, Szabo G, Lele Z: Expression of two type II cadherins, Cdh12 and Cdh22 in the developing and adult mouse brain. Gene Expr Patterns 2010, 10:351-360.

10. Bankovic J, Stojsic J, Jovanovic D, Andjelkovic T, Milinkovic V, Ruzdijic S, Tanic N: Identification of genes associated with non-small-cell lung cancer promotion and progression. Lung Cancer 2010, 67:151-159.
11. Wang JF, She L, Su BH, Ding LC, Zheng FF, Zheng DL, Lu YG: CDH12 promotes the invasion of salivary adenoid cystic carcinoma. Oncol Rep 2011, 26:101-108.

12. Hu H, Krasinskas A, Willis J: Perspectives on current tumor-node-metastasis (TNM) staging of cancers of the colon and rectum. Semin Oncol 2011, 38:500-510.

13. Shimazui T, Kojima T, Onozawa M, Suzuki M, Asano T, Akaza H: Expression profile of $\mathrm{N}$-cadherin differs from other classical cadherins as a prognostic marker in renal cell carcinoma. Oncol Rep 2006, 15:1181-1184.

14. Zhan DQ, Wei S, Liu C, Liang BY, Ji GB, Chen XP, Xiong M, Huang ZY: Reduced $\mathrm{N}$-cadherin expression is associated with metastatic potential and poor surgical outcomes of hepatocellular carcinoma. J Gastroenterol Hepatol 2012, 27:173-180.

15. Li P, Lin Y, Zhang Y, Zhu Z, Huo K: SSX2IP promotes metastasis and chemotherapeutic resistance of hepatocellular carcinoma. J Trans/ Med 2013, 11:52.

16. Li P, Chen X, Su L, Li C, Zhi Q, Yu B, Sheng H, Wang J, Feng R, Cai Q, et al: Epigenetic silencing of miR-338-3p contributes to tumorigenicity in gastric cancer by targeting SSX2IP. PLoS One 2013, 8:e66782.

17. Mirshahi P, Rafii A, Vincent L, Berthaut A, Varin R, Kalantar G, Marzac C, Calandini OA, Marie JP, Soria C, et al: Vasculogenic mimicry of acute leukemic bone marrow stromal cells. Leukemia 2009, 23:1039-1048.

18. Natalwala A, Spychal R, Tselepis C: Epithelial-mesenchymal transition mediated tumourigenesis in the gastrointestinal tract. World J Gastroenterol 2008, 14:3792-3797.

19. Valastyan S, Weinberg RA: Tumor metastasis: molecular insights and evolving paradigms. Cell 2011, 147:275-292.

20. Hanahan D, Weinberg RA: Hallmarks of cancer: the next generation. Cell 2011, 144:646-674.

doi:10.1186/1479-5876-11-288

Cite this article as: Zhao et al:: Cadherin-12 contributes to

tumorigenicity in colorectal cancer by promoting migration, invasion, adhersion and angiogenesis. Journal of Translational Medicine 2013 11:288.

\section{Submit your next manuscript to BioMed Central and take full advantage of:}

- Convenient online submission

- Thorough peer review

- No space constraints or color figure charges

- Immediate publication on acceptance

- Inclusion in PubMed, CAS, Scopus and Google Scholar

- Research which is freely available for redistribution 